\title{
Wind Accretion - Observations Vs Theory
}

\section{N. Shakura*}

Sternberg Astronomical Institute, Moscow M.V.Lomonosov State University

E-mail: nikolai.shakura@gmail.com

\section{K. Postnov}

Sternberg Astronomical Institute, Moscow M.V.Lomonosov State University

E-mail: kpostnovegmail.com

The theory of quasi-spherical subsonic accretion onto magnetized rotating neutron star is reviewed. Different regimes of quasi-spherical accretion onto a neutron star: supersonic (Bondi) accretion, which takes place when the captured matter cools down rapidly and falls supersonically towards the neutron-star magnetosphere, and subsonic (settling) accretion which occurs when the plasma remains hot until it meets the magnetospheric boundary. In subsonic accretion, which works at X-ray luminosities $\lesssim 4 \times 10^{36} \mathrm{erg} \mathrm{s}^{-1}$, a hot quasi-spherical shell must form around the magnetosphere, and the actual accretion rate onto the neutron star is determined by the ability of the plasma to enter the magnetosphere due to the Rayleigh-Taylor instability. We show how the dimensionless parameters of the theory can be determined from observations of equilibrium X-ray pulsars (Vela X-1, GX 301-2). We also discuss how in the settling accretion theory bright $\mathrm{X}$-ray flares $\left(\sim 10^{38}-10^{40} \mathrm{ergs}\right)$ observed in supergiant fast X-ray transients (SFXT) may be produced by sporadic capture of magnetized stellar-wind plasma. At sufficiently low accretion rates, magnetic reconnection can enhance the magnetospheric plasma entry rate, resulting in copious production of X-ray photons, strong Compton cooling and ultimately in unstable accretion of the entire shell. A bright flare develops on the free-fall time scale in the shell, and the typical energy released in an SFXT bright flare corresponds to the mass of the shell.

Accretion Processes in Cosmic Sources - APCS2016

5-10 September 2016

Saint Petersburg, Russia

\footnotetext{
${ }^{*}$ Speaker.
} 


\section{Introduction: Short history of $X$-ray astronomy and accretion}

On June 18, 1962, a serendipitous discovery of the first galactic X-ray source, Sco X-1, was made [1]. The project was originally aimed at observing X-ray fluorescent emission from the Moon, but instead this discovery heralded the beginning of X-ray astronomy. The fluorescent Xray emission from the Moon was actually discovered about 20 years later by the ROSAT satellite [2]. Sco X-1 was the brightest galactic X-ray source far beyond the Solar system, and later a lot of interesting galactic X-ray sources (Cyg X-1, Her X-1, Cen X-3, etc.) were discovered in other rocket experiments. Before the launch of the specialized X-ray satellite UHURU (12 December 1970, [3]), the origin of the powerful X-ray emission from galactic sources was unclear. However, as early as in the mid-1960, Yakov Zeldovich [4] and Ed Salpeter [5] invoked accretion of matter onto moving compact objects as powerful source of energy emission. First UHURU results showed that galactic X-ray sources can be quasi-persistent (like Cyg X-1 [6]) or show periodic pulsations (like Cen X-3 [7] and Her X-1 [8]). Later it was recognized that disk accretion onto a compact star in a binary system is responsible for the observed powerful X-ray emission [9]. In close binary systems, accretion disks are formed during mass transfer from the optical star onto compact stellar remnants (neutron stars or black holes) through the vicinity of the inner Lagrangian point. In the case of black holes, accretion disks extend down the to the last marginally stable circular orbit $\left(6 G M / c^{2}\right.$ for a Schwarzschild black hole). In the case of magnetized neutron stars, the magnetic field of neutron star starts destroying accretion flow at distances typically about 100-1000 NS radii. The accreting matter enters the NS magnetosphere, gets frozen into the NS magnetic field and is canalized to the NS magnetic polar caps, where most of the accretion power is emitted. The disk accretion regime is usually realized when the optical star overfills its Roche lobe. If the optical star does not fill its Roche lobe, accretion still can be very powerful from the captured stellar wind $[9,10]$. Even in this case accretion disk can be formed if the specific angular momentum of captured matter is high enough; if not, accretion flow will be quasi-spherical. In this review we will consider only quasi-spherical accretion onto magnetized NSs.

\section{Two regimes of wind accretion}

Quasi-spherical accretion is most likely to occur in high-mass X-ray binaries (HMXB) when the optical star of early spectral class (OB) does not fill its Roche lobe, but experiences a significant mass loss via stellar wind. We shall discuss the wind accretion regime, in which a bow shock forms in the stellar wind around the compact star. The characteristic distance at which the bow shock forms is about the gravitational capture (Bondi) radius

$$
R_{B}=2 G M /\left(v_{w}^{2}+v_{o r b}^{2}\right)
$$

where $v_{w}$ is the wind velocity (typically $100-1000 \mathrm{~km} / \mathrm{s}$ ), $v_{\text {orb }}$ is the orbital velocity of NS, which is usually much smaller than $v_{w}$, so below we will neglect it. The rate of gravitational capture of mass from the wind with density $\rho_{w}$ near the orbital position of the NS is the Bondi-Hoyle-Littleton mass accretion rate:

$$
\dot{M}_{B} \simeq \rho_{w} R_{B}^{2} v_{w} \propto \rho_{w} v_{w}^{-3} .
$$




\subsection{Supersonic (Bondi-Hoyle-Littleton) accretion}

There can be two different cases of quasi-spherical accretion. The classical Bondi-HoyleLittleton accretion takes place when the shocked matter rapidly cools down, and the matter freely falls towards the NS magnetosphere (see Fig. 1) by forming a shock at some distance above the magnetosphere. Here the shocked matter cools down (mainly by Compton processes) and enters the magnetosphere via Rayleigh-Taylor instability [11]. The magnetospheric boundary is characterized by the Alfvén radius $R_{A}$, which can be calculated from the balance of the ram pressure of the infalling matter and the magnetic field pressure at the magnetospheric boundary: $\rho v_{f f}^{2}\left(R_{A}\right)=B^{2} / 8 \pi$. Making use of the mass continuity equation in the shell, $\dot{M}=4 \pi R^{2} \rho(R) v_{f f}(R)$, and assuming dipole NS magnetic field, the standard result [10] is obtained:

$$
R_{A}=\left(\frac{\mu^{2}}{\dot{M} \sqrt{2 G M}}\right)^{2 / 7} .
$$

The captured matter from the wind carries a specific angular momentum $j_{w} \sim \omega_{B} R_{B}^{2}$ [12]. Depending on the sign of $j_{w}$ (prograde or retorgrade), the NS can spin-up or spin-down. This regime of quasi-spherical accretion occurs in bright X-ray pulsars with $L_{x}>4 \times 10^{36} \mathrm{erg} \mathrm{s}^{-1}[13,14]$.

\subsection{Subsonic (settling) accretion}

If the captured wind matter behind the bow shock at $R_{B}$ remains hot (when the plasma cooling time is much longer than the free-fall time, $t_{\text {cool }} \gg t_{f f}$ ), a hot quasi-static shell forms around the magnetosphere. The subsonic (settling) accretion sets in (see Fig. 2). In this case, both spin-up or spin-down of the NS is possible, even if the sign of $j_{w}$ is positive (prograde). The shell mediates the angular momentum transfer from the NS magnetosphere via viscous stresses due to convection and turbulence. In this regime, the mean radial velocity of matter in the shell $u_{r}$ is smaller than the free-fall velocity $u_{f f}: u_{r}=f(u) u_{f f}, f(u)<1$, and is determined by the plasma cooling rate near the magnetosphere (due to the Compton or radiative cooling):

$$
f(u) \sim\left[t_{f f}\left(R_{A}\right) / t_{c o o l}\left(R_{A}\right)\right]^{1 / 3} .
$$

In the settling accretion regime the actual mass accretion rate onto NS can be significantly smaller than the Bondi mass accretion rate,

$$
\dot{M}=f(u) \dot{M}_{B}
$$

The settling accretion occurs at $L_{x}<4 \times 10^{36} \mathrm{erg} \mathrm{s}^{-1}$ [14].

\section{Structure of the shell}

The structure of the shell around NS magnetosphere in the settling accretion regime is discussed in detail in [14]. To the first approximation, its vertical structure along the radius $R$ can be described assuming hydrostatic equilibrium:

$$
-\frac{1}{\rho} \frac{d P}{d R}=\frac{G M}{R^{2}}
$$




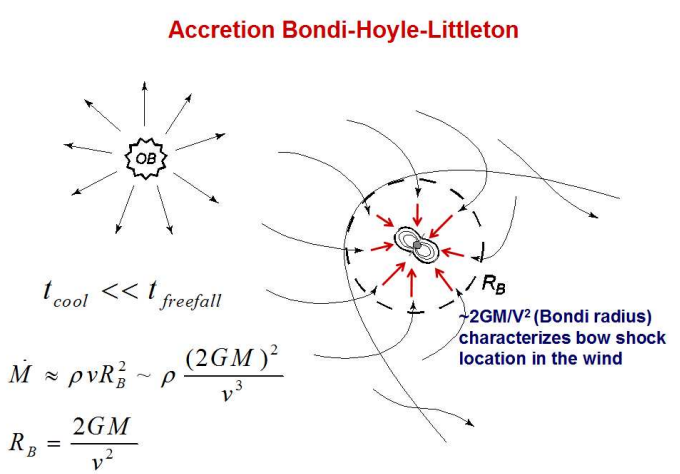

Figure 1: Supersonic (Bondi-Hoyle-Littleton) accretion onto magnetized NS

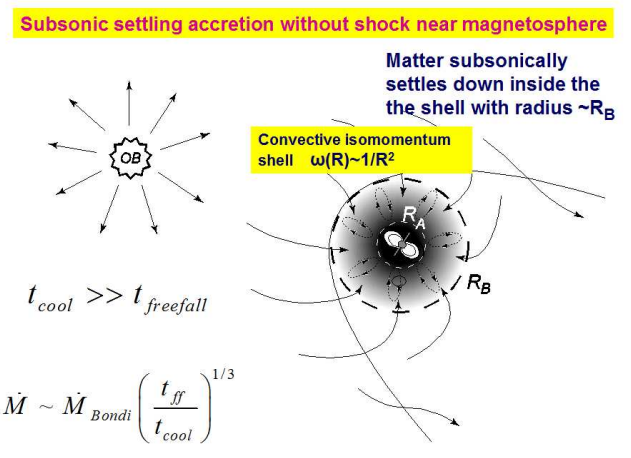

Figure 2: Subsonic settling accretion onto magnetized NS

with the adiabatic solution for the temperature radial profile

$$
\frac{R T}{\mu_{m}}=\frac{\gamma-1}{\gamma} \frac{G M}{R} .
$$

For the adiabatic index $\gamma=5 / 3$ we get the standard result (see also [15]):

$$
\rho(R)=\rho\left(R_{A}\right)\left(\frac{R_{A}}{R}\right)^{3 / 2}
$$

where $R_{A}$ is the magnetospheric (Alfv'en) radius.

Unlike the supersonic Bondi regime, in the settling accretion regime the magnetospheric boundary is determined by balance between the gas thermal pressure and magnetic pressure yielding [14]

$$
R_{A}=\left[\frac{4 \gamma}{\gamma-1} f(u) K_{2} \frac{\mu^{2}}{\dot{M} \sqrt{2 G M}}\right]^{2 / 7},
$$

where the factor $K_{2} \simeq 7.6$ takes into account the effect of magnetospheric currents [11]. Clearly, in the settling accretion regime the dependence of $R_{A}$ on $\dot{M}$ and $\mu$ can be different than in the standard formula Eq. (2.3), since the factor $f(u)$ depends differently on $\dot{M}$ and $\mu$ for different cooling regime. Numerically, $f(u) \sim 0.1-0.5$ depending on the X-ray luminosity. The plasma enters the magnetosphere of the slowly rotating neutron star due to the Rayleigh-Taylor instability. The boundary between the plasma and the magnetosphere is stable at high temperatures $T>T_{c r}$, but becomes unstable at $T<T_{c r}$, and remains in a neutral equilibrium at $T=T_{c r}$ [16]. The critical temperature is:

$$
\mathcal{R} T_{c r}=\frac{1}{2} \frac{\cos \chi}{\kappa R_{A}} \frac{\mu_{m} G M}{R_{A}} .
$$

Here $\kappa$ is the local curvature of the magnetosphere, $\chi$ is the angle the outer normal to the magnetospheric surface makes with the radius-vector at a given point. The effective gravity acceleration can be written as

$$
g_{\text {eff }}=\frac{G M}{R_{A}^{2}} \cos \chi\left(1-\frac{T}{T_{c r}}\right) .
$$


The temperature in the quasi-static shell is given by Eq. (3.2), and the condition for the magnetosphere instability can thus be rewritten as:

$$
\frac{T}{T_{c r}}=\frac{2(\gamma-1)}{\gamma} \frac{\kappa R_{A}}{\cos \chi}<1
$$

Consider, for example, the development of the interchange instability when cooling (predominantly Compton cooling) is present. The temperature changes as [17], [18]

$$
\frac{d T}{d t}=-\frac{T-T_{x}}{t_{C}}
$$

where the Compton cooling time is

$$
t_{C}=\frac{3}{2 \mu_{m}} \frac{\pi R_{A}^{2} m_{e} c^{2}}{\sigma_{T} L_{x}} \approx 10.6[\mathrm{~s}] R_{9}^{2} \dot{M}_{16}^{-1} .
$$

Here $m_{e}$ is the electron mass, $\sigma_{T}$ is the Thomson cross section, $L_{x}=0.1 \dot{M} c^{2}$ is the X-ray luminosity, $T$ is the electron temperature (which is equal to the ion temperature since the timescale of electronion energy exchange here is the shortest possible), $T_{x}$ is the X-ray temperature and $\mu_{m}=0.6$ is the molecular weight. The photon temperature is $T_{x}=(1 / 4) T_{\text {cut }}$ for a bremsstrahlung spectrum with an exponential cut-off at $T_{c u t}$, typically $T_{x}=3-5 \mathrm{keV}$. The solution of equation Eq. (3.8) reads:

$$
T=T_{x}+\left(T_{c r}-T_{x}\right) e^{-t / t_{C}}
$$

We note that $T_{c r} \sim 30 \mathrm{keV} \gg T_{x} \sim 3 \mathrm{keV}$. It is seen that for $t \approx 2 t_{C}$ the temperature decreases to $T_{x}$. In the linear approximation the temperature changes as:

$$
T \approx T_{c r}\left(1-t / t_{C}\right)
$$

Plugging this expression into Eq. (3.6), we find that the effective gravity acceleration increases linearly with time as:

$$
g_{\text {eff }} \approx \frac{G M}{R_{A}^{2}} \frac{t}{t_{C}} \cos \chi .
$$

Correspondingly, the velocity of matter due to the instability growth increases with time as:

$$
u_{i}=\int_{0}^{t} g_{e f f} d t=\frac{G M}{R_{A}^{2}} \frac{t^{2}}{2 t_{C}} \cos \chi .
$$

Let us introduce the mean rate of the instability growth

$$
<u_{i}>=\frac{\int u d t}{t}=\frac{1}{6} \frac{G M}{R_{A}^{2}} \frac{t^{2}}{t_{C}}=\frac{1}{6} \frac{G M}{R_{A}^{2} t_{C}}\left(\frac{\zeta R_{A}}{<u_{i}>}\right)^{2} \cos \chi .
$$

Here $\zeta \lesssim 1$ and $\zeta R_{A}$ is the characteristic scale of the instability that grows with the rate $\left\langle u_{i}>\right.$. Therefore, for the mean rate of the instability growth at the linear stage we find

$$
<u_{i}>=\left(\frac{\zeta^{2} G M}{6 t_{C}}\right)^{1 / 3}=\frac{\zeta^{2 / 3}}{12^{1 / 3}} \sqrt{\frac{2 G M}{R_{A}}}\left(\frac{t_{f f}}{t_{C}}\right)^{1 / 3} \cos \chi
$$


As the factor $\cos \chi \simeq 1$, we will omit it below. Here we have introduced the characteristic time as

$$
t_{f f}=\frac{R_{A}^{3 / 2}}{\sqrt{2 G M}},
$$

which is close to the free-fall time at a given radius. Therefore, the factor $f(u)$ becomes:

$$
f(u)=\frac{<u_{i}>}{u_{f f}\left(R_{A}\right)}
$$

Substituting Eq. (3.15) and Eq. (3.17) into Eq. (3.4), we find for the Alfvén radius in this regime:

$$
R_{A}^{(C)} \approx 1.37 \times 10^{9}[\mathrm{~cm}]\left(\zeta \frac{\mu_{30}^{3}}{\dot{M}_{16}}\right)^{2 / 11}
$$

Plugging Eq. (3.18) into Eq. (3.17), we obtain the explicit expression for $f(u)$ in the Compton cooling regime:

$$
f(u)_{C} \approx 0.22 \zeta^{7 / 11} \dot{M}_{16}^{4 / 11} \mu_{30}^{-1 / 11}
$$

In the radiation cooling regime the radiation cooling time is

$$
t_{\text {cool }}^{(r a d)}=\frac{3 k T}{2 \mu_{m} n_{e} \Lambda(T)}=\sqrt{T} / K_{\text {rad }}
$$

where $\Lambda(T) \approx 2.5 \times 10^{-27} \sqrt{T}$ (in CGS units) is the radiation cooling factor (here the Gaunt-factor is taken into account and that real cooling function at high temperatures goes slightly higher than for pure free-free emission). With this cooling time, temperature decreases as

$$
\frac{d T}{d t}=-K_{\text {rad }} \sqrt{T}
$$

yielding a non-exponential temperature decay with time

$$
\frac{T}{T_{0}}=\left(1-\frac{1}{2} \frac{K_{r a d} t}{\sqrt{T_{0}}}\right)^{2}
$$

In the linear approximation, when $t \ll t_{\text {cool }}^{(\mathrm{rad})}$, we get for the radiation cooling law

$$
\frac{T}{T_{c r}}=1-\frac{t}{t_{\text {cool }}^{(r a d)}}
$$

similarly to Eq. (3.11) for Compton cooling, and find

$$
\begin{gathered}
R_{A}^{(r a d)} \approx 1.05 \times 10^{9}[\mathrm{~cm}] \zeta^{4 / 81} \mu_{30}^{16 / 27} \dot{M}_{16}^{-6 / 27} \\
f(u)_{r a d} \approx 0.1 \zeta^{14 / 81} \mu_{30}^{2 / 27} \dot{M}_{16}^{6 / 27}
\end{gathered}
$$




\section{Spin-up/spin-down of neutron star during settling accretion}

At the settling accretion stage onto a NS in a binary system, there are three characteristic angular frequencies: the angular orbital frequency $\omega_{b}=2 \pi / P_{b}$, which characterizes the specific angular momentum of captured matter, the angular frequency of matter near the magnetosphere, $\omega_{m}\left(R_{A}\right)$, and the angular frequency of magnetosphere $\omega^{*}=2 \pi / P^{*}$ which coincides with the NS spin frequency. If $\omega_{m}\left(R_{A}\right)-\omega^{*} \neq 0$, an effective exchange of angular momentum between the magnetosphere and the quasi-spherical shell occurs. As shown in Appendices in [14], [19], the rotational law in the shell with settling accretion can be represented in a power-law from $\omega(R) \sim$ $1 / R^{n}$, with $0 \leq n \leq 2$ depending on the treatment of viscous stresses $W_{R \phi}$ in the shell. In the most likely case where anisotropic turbulence appears due to near-sonic convection (see [14]), $n \approx 2$, i.e. iso-angular-momentum rotational law sets in.

The torque due to magnetic forces applied to the neutron star reads:

$$
I \dot{\omega}^{*}=\int \frac{B_{t} B_{p}}{4 \pi} \varpi d S
$$

where $B_{t}$ is the toroidal magnetic field component which arises if there is the difference of the angular velocity of matter $\omega_{m}$ and magnetosphere angular rotation $\omega^{*}$. On the other hand, there is a mechanical torque on the magnetosphere from the base of the shell caused by the turbulent stresses $W_{R \phi}$ :

$$
\int W_{R \phi} \varpi d S
$$

where the viscous turbulent stresses can be written as

$$
W_{R \phi}=\rho v_{t} R \frac{\partial \omega}{\partial R} .
$$

To specify the turbulent viscosity coefficient

$$
v_{t}=\left\langle u_{c} l_{t}\right\rangle
$$

we assume that the characteristic scale of the turbulence close to the magnetosphere is

$$
l_{t}=\zeta_{d} R_{A},
$$

where we have introduced the dimensionless factor $\zeta_{d} \lesssim 1$, characterizing the size of the zone in which there is an effective exchange of angular momentum between the magnetosphere and the base of the shell. The characteristic velocity of the turbulent pulsations $u_{c}$ is determined by the mechanism of turbulence in the plasma above the magnetosphere. In the case of strong convective motions in the shell, caused by heating of its base, $u_{c} \sim c_{s}$, where $c_{s}$ is the sound speed. Equating the torques Eq. (4.1) and Eq. (4.2) and allowing for Eq. (4.3) and Eq. (4.5), we get

$$
\rho u_{c} \zeta_{d} R_{A}^{2} \frac{\partial \omega}{\partial R}=\frac{B_{t} B_{p}}{4 \pi}
$$

We eliminate the density from this expression using the pressure balance at the magnetospheric boundary and the expression for the temperature Eq. (3.2), and make the substitution

$$
\frac{\partial \omega}{\partial R}=\frac{\omega_{m}-\omega^{*}}{\zeta_{d} R_{A}}
$$


Then we find the relation between the toroidal and poloidal components of the magnetic field in the magnetosphere:

$$
\frac{B_{t}}{B_{p}}=K_{2} \frac{\gamma}{\sqrt{2}(\gamma-1)}\left(\frac{u_{c}}{u_{f f}}\right)\left(\frac{\omega_{m}-\omega^{*}}{\omega_{K}\left(R_{A}\right)}\right) .
$$

(Note that there is no dependence on the width of the layer characterized by the parameter $\zeta_{d}$ ). Substituting Eq. (4.8) into Eq. (4.1), the spin-down rate of the neutron star can be written as:

$$
I \dot{\omega}^{*}=K_{1} K_{2}\left(\frac{u_{c}}{u_{f f}}\right) \frac{\mu^{2}}{R_{A}^{3}} \frac{\omega_{m}-\omega^{*}}{\omega_{K}\left(R_{A}\right)} .
$$

where $K_{1} \sim 1$ is a constant arising from integrating of torques over the magnetospheric surface.

Using the definition of the Alfvén radius $R_{A}$ Eq. (3.4) and the expression for the Keplerian frequency $\omega_{K}$, we can write Eq. (4.9) in the form

$$
I \dot{\omega}^{*}=Z \dot{M} R_{A}^{2}\left(\omega_{m}-\omega^{*}\right) .
$$

Here the dimensionless coefficient $Z$ is

$$
Z=K_{1}\left(\frac{u_{c}}{u_{f f}}\right) \frac{1}{f(u)}
$$

Taking into account that the matter falling onto the neutron star adds the angular momentum $z \dot{M} R_{A}^{2} \omega^{*}$, we ultimately get

$$
I \dot{\omega}^{*}=Z \dot{M} R_{A}^{2}\left(\omega_{m}-\omega^{*}\right)+z \dot{M} R_{A}^{2} \omega^{*} .
$$

Here $0<z<1$ is the numerical coefficient which is $\sim 2 / 3$ if matter enters across the magnetospheric surface with equal probability at different magnetospheric latitudes. Substituting $\omega_{m}\left(R_{A}\right)=$ $\omega_{B}\left(R_{B} / R_{A}\right)^{2}$ for iso-angular-momentum shell, we can rewrite the above equation in the form

$$
I \dot{\omega}^{*}=Z \dot{M} \omega_{B} R_{B}^{2}-Z(1-z / Z) \dot{M} R_{A}^{2} \omega^{*} .
$$

Substituting for the coupling coefficient $Z$, in the case of Compton cooling we can rewrite Eq. (4.12) in the form explicitly showing the spin-up $\left(K_{s u}\right)$ and spin-down $\left(K_{s d}\right)$ torques:

$$
\dot{\omega}^{*}=A \dot{M}^{\frac{7}{11}}-B \dot{M}^{3 / 11}=K_{s u}-K_{s d} .
$$

Here the spin-up/spin-down coefficients $A$ and $B$ do not explicitly depend on $\dot{M}$.

For a characteristic value of the accretion rate $\dot{M}_{16} \equiv \dot{M} / 10^{16} \mathrm{~g} / \mathrm{s}$, the spin-up and spin-down torques read (in CGS units):

$$
\begin{aligned}
K_{s u} \approx 5.29 \times 10^{-13}\left[\mathrm{rad} / \mathrm{s}^{2}\right] K_{1}\left(\frac{u_{c}}{u_{f f}}\right) \zeta^{-\frac{7}{11}} \mu_{30}^{\frac{1}{11}}\left(\frac{v_{8}}{\sqrt{\delta}}\right)^{-4}\left(\frac{P_{b}}{10 \mathrm{~d}}\right)^{-1} \dot{M}_{16}^{7 / 11} I_{45}^{-1} \\
K_{s d} \approx 5.36 \times 10^{-12}\left[\mathrm{rad} / \mathrm{s}^{2}\right](1-z / Z) K_{1}\left(\frac{u_{c}}{u_{f f}}\right) \zeta^{-3 / 11} \mu_{30}^{13 / 11}\left(\frac{P^{*}}{100 \mathrm{~s}}\right)^{-1} \dot{M}_{16}^{3 / 11} I_{45}^{-1} .
\end{aligned}
$$

Here $I_{45}=I / 10^{45} \mathrm{~g} \mathrm{~cm}^{2}$ is the NS moment of inertia, the dimensionless factor $\delta \sim 1$ takes into account the actual location of the gravitational capture radius.

Another approach to the problem of interaction of quasi-spherically accreting magnetized plasma with rotating NS magnetospheres is developed in [20]. 


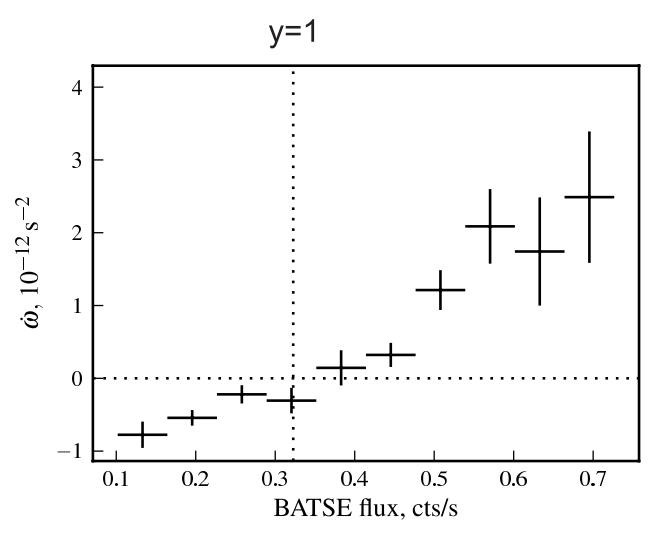

Figure 3: Torque-luminosity correlation in GX $301-2, \dot{\omega}^{*}$ as a function of BATSE data $(20-40 \mathrm{keV}$ pulsed flux) near the equilibrium frequency [21]. The assumed X-ray flux at equilibrium (in terms of the dimensionless parameter $y$ ) is also shown by the vertical dotted line.

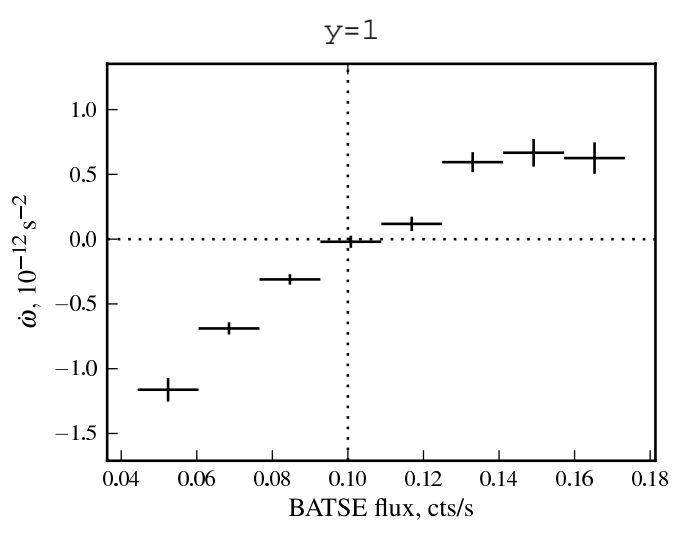

Figure 4: The same as in Fig. 3 for Vela X-1 (V.Doroshenko, PhD Thesis, 2010, IAAT)

\section{Equilibrium pulsars}

For equilibrium pulsars we set $\dot{\omega}^{*}=0$ and from Equation Eq. (4.12) we get

$$
Z_{e q}\left(\omega_{m}-\omega^{*}\right)+z \omega^{*}=0 .
$$

Close to equilibrium we may vary Eq. (4.12) with respect to $\dot{M}$. Variations in $\delta \dot{M}$ may in general be caused by changes in density $\delta \rho$ as well as in velocity of the stellar wind $\delta v$ (and thus the Bondi radius). For density variations only we find (see Eq. (67) in [22] for more detail)

$$
Z_{e q, \rho}=\frac{\left.I \frac{\partial \dot{\omega}^{*}}{\partial \dot{M}}\right|_{e q}}{\frac{4}{11} \omega^{*} R_{A}^{2}} \approx 2.52\left(\frac{\left.\frac{\partial \dot{\omega}^{*}}{\partial y}\right|_{y=1}}{10^{-12}}\right)\left(\frac{P^{*}}{100 s}\right) \zeta^{-4 / 11} \dot{M}_{16}^{-7 / 11} \mu_{30}^{-12 / 11}
$$

On the other hand, by equating this value to the definition of the coupling coefficient $Z$ (see Eq. (4.11) above), we can find the dimensionless combination of the theory parameters:

$$
\Pi_{0} \equiv \frac{K_{1}\left(\frac{u_{c}}{u_{f f}}\right)}{\zeta^{3 / 11}} \approx 0.55\left(\frac{\left.\frac{\partial \dot{\omega}^{*}}{\partial y}\right|_{y=1}}{10^{-12}}\right)\left(\frac{P^{*}}{100 s}\right) \dot{M}_{16}^{-3 / 11} \mu_{30}^{-13 / 11}
$$

The equilibrium period of an X-ray pulsar with known NS magnetic field can be found from Eq. (4.13) (or, which is the same, by equating the spin-up and spin-down torques from Eq. (4.15) and eq. (4.16)):

$$
P_{e q} \approx 1000[\mathrm{~s}]\left(1-z / Z_{e q}\right) \zeta^{4 / 11} \mu_{30, e q}^{12 / 11}\left(\frac{P_{b}}{10 \mathrm{~d}}\right) \dot{M}_{16}^{-4 / 11}\left(\frac{v_{8}}{\sqrt{\delta}}\right)^{4}
$$


In the equilibrium, from this formula we can determine another dimensionless combination of the theory parameters:

$$
\Pi_{1} \equiv \frac{\left(1-\frac{z}{Z_{e q}}\right) \zeta^{4 / 11}}{\delta^{2}} \approx 0.1\left(\frac{P^{*}}{100 s}\right)\left(\frac{P_{b}}{10 \mathrm{~d}}\right)^{-1} \dot{M}_{16}^{4 / 11} \mu_{30}^{-12 / 11} v_{8}^{-4} .
$$

Because of the strong dependence of the equilibrium period on (usually, poorly measurable) wind velocity, for pulsars with independently known magnetic fields $\mu$ it is more convenient to estimate the wind velocity, assuming $P^{*}=P_{e q}^{*}$ :

$$
v_{8} \approx 0.56\left[\frac{\left(1-\frac{z}{Z_{e q}}\right) \zeta^{4 / 11}}{\delta^{2}}\right]^{-1 / 4} \dot{M}_{16}^{1 / 11} \mu_{30, e q}^{-3 / 11}\left(\frac{P_{*} / 100 \mathrm{~s}}{P_{b} / 10 \mathrm{~d}}\right)^{1 / 4}
$$

which is only weakly dependent on $\dot{M}$ and the theory parameter $\Pi_{1}$.

In the possible case of mass accretion rate variations due to wind velocity changes only, the coupling coefficient $Z_{e q, v}$ reads (see Eq. (68) in [22]):

$$
Z_{e q, v} \approx 0.76\left(\frac{\left.\frac{\partial \dot{\omega}^{*}}{\partial y}\right|_{y=1}}{10^{-12}}\right)\left(\frac{P^{*}}{100 s}\right) \zeta^{-4 / 11} \dot{M}_{16}^{-7 / 11} \mu_{30}^{-12 / 11}+\frac{7}{10} z
$$

Clearly, in this case the coupling is smaller. Below we will consider only wind density variations. In principle, if $z>0$ and $\left(\omega_{m}-\omega^{*}\right)>0$, Eq. (5.1) implies that there can be no equilibrium at all the pulsar can only spin-up. However, two well-measured equilibrium pulsars (see below) show that the equilibrium does exist, suggesting that in these objects $\left(\omega_{m}-\omega^{*}\right)<0$.

To illustrate the theory outlined above, we show the measured and obtained model parameters of two well-known persistent X-ray puslars, Vela X-1 and GX 301-2 (see Table 5).

It is clear from Table 5 that for Vela X-1 observed and derived parameters are in good agreement, with the value of dimensionless theory parameters $\Pi_{0} \sim 1$, as expected from very general hydrodynamic similarity principles [23]. It is remarkable that parameter $\Pi_{0} \sim 1$ in GX 301-2 as well, suggesting the common physics of hydrodynamic interactions in these objects. However, the observed wind velocity in GX 301-2 is inferred from observations to be around $300 \mathrm{~km} / \mathrm{s}$, which is almost two times as small as derived from our theory. To obtain such a low velocity from Eq. (5.6), the dimensionless parameter $\Pi_{1}$ should be around 10, which is unrealistically high (in fact, this parameter should not be higher than 1). From this we conclude that in GX 301-2 the wind velocity is likely to be estimated not close to the interaction region with NS.

\section{Non-equilibrium pulsars}

It is convenient to introduce the dimensionless parameter

$$
y \equiv \frac{\dot{M}}{\dot{M}_{e q}}
$$

where $\dot{M}_{e q}$ represents the accretion rate at which $\dot{\omega}^{*}=0$ :

$$
\dot{M}_{e q}=\left(\frac{B}{A}\right)^{11 / 4} \text {. }
$$


Table 1: Parameters for the equilibrium X-ray pulsars.

\begin{tabular}{lcc}
\hline Pulsar & \multicolumn{3}{c}{ Equilibrium pulsars } \\
\hline \multicolumn{3}{c}{ GX301-2 VelaX -1 } \\
\hline \multicolumn{3}{c}{ Measured parameters } \\
\hline$P^{*}(\mathrm{~s})$ & 680 & 283 \\
$P_{B}(\mathrm{~d})$ & 41.5 & 8.96 \\
$v_{w}(\mathrm{~km} / \mathrm{s})$ & $300 ?$ & 700 \\
$\mu_{30}$ & 2.7 & 1.2 \\
$\dot{M}_{16}$ & 3 & 3 \\
$\left.\frac{\partial \dot{\omega}}{\partial y}\right|_{y=1}\left(\mathrm{rad} / \mathrm{s}^{2}\right)$ & $1.5 \cdot 10^{-12}$ & $1.2 \cdot 10^{-12}$ \\
\hline \multicolumn{3}{c}{ Derived parameters } \\
\hline$f(u) \zeta^{-7 / 11}$ & 0.32 & 0.30 \\
$Z_{e q} \zeta^{4 / 11}$ & 4.32 & 3.49 \\
$\Pi_{0}$ & 1.28 & 1.11 \\
$v_{8} \Pi_{1}^{1 / 4}(\mathrm{~km} / \mathrm{s})$ & 530 & 800 \\
\hline \multicolumn{3}{c}{}
\end{tabular}

Equation Eq. (4.14) can be rewritten in the form

$$
I \dot{\omega}^{*}=A \dot{M}_{e q}^{\frac{7}{11}} y^{\frac{7}{11}}\left(1-y^{-\frac{4}{11}}\right) .
$$

The plot of the function $\dot{\omega}^{*}(y)$ is shown schematically in Fig. 5. The function $\dot{\omega}^{*}(\dot{M})$ reaches minimum at $\dot{M}=\dot{M}_{c r}$ :

$$
\dot{M}_{c r}=\dot{M}_{e q}\left(\frac{3}{7}\right)^{\frac{11}{4}},
$$

In other words, $\dot{\omega}^{*}$ attains minimum for the dimensionless parameter

$$
y_{c r}=\left(\frac{3}{7}\right)^{\frac{11}{4}}<1
$$

The minimum $\dot{\omega}^{*}$ for $y=y_{c r}$ (i.e. the maximum possible spin-down rate of the pulsar) is

$$
I \dot{\omega}_{\min }^{*}=-\frac{4}{3} A \dot{M}_{e q}^{\frac{7}{11}} y^{\frac{7}{11}}
$$

Numerically, the maximum spin-down rate at $y_{c r}$ is

$$
\dot{\omega}_{s d, \min }^{*} \approx-1.12 \times 10^{-12}\left[\mathrm{rad} / \mathrm{s}^{2}\right](1-z / Z)^{7 / 4} K_{1}\left(\frac{u_{c}}{u_{f f}}\right) \mu_{30}^{2}\left(\frac{v_{8}}{\sqrt{\delta}}\right)^{3}\left(\frac{P^{*}}{100 \mathrm{~s}}\right)^{-7 / 4}\left(\frac{P_{b}}{10 \mathrm{~d}}\right)^{3 / 4} .
$$

Then, from the condition $\left|\dot{\omega}_{s d}^{*}\right| \leq\left|\dot{\omega}_{s d, \text { min }}^{*}\right|$ follows a lower limit on the neutron star magnetic field:

$$
\mu_{30}>\mu_{30, \text { min }}^{\prime} \approx 0.94\left|\frac{\dot{\omega}_{s d}^{*}}{10^{-12} \mathrm{rad} / \mathrm{s}^{2}}\right|^{1 / 2}(1-z / Z)^{-7 / 8}\left(K_{1}\left(\frac{u_{c}}{u_{f f}}\right)\right)^{-1 / 2}\left(\frac{v_{8}}{\sqrt{\delta}}\right)^{-3 / 2}\left(\frac{P^{*}}{100 \mathrm{~s}}\right)^{7 / 8}\left(\frac{P_{b}}{10 \mathrm{~d}}\right)^{-3 / 8} .
$$




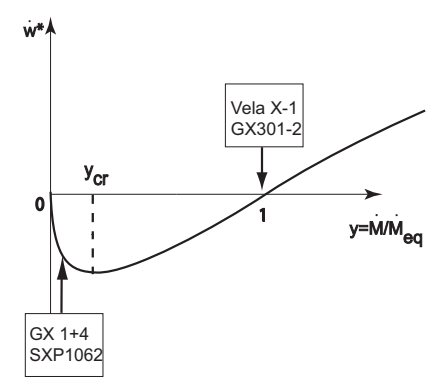

Figure 5: Schematics of the dependence of $\dot{\omega}^{*}$ on the dimensionless accretion rate $y$. The figure shows the position in the diagram for equilibrium pulsars with $y \sim 1$ and for non-equilibrium pulsars at steady spin-down with $y<y_{c r}$

Table 2:

\begin{tabular}{lccc}
\hline Pulsars & \multicolumn{3}{c}{ Gon-equilibrium pulsars } \\
\hline \multicolumn{4}{c}{ Measured parameters } \\
\hline$P^{*}(\mathrm{~s})$ & 140 & 1062 & 5560 \\
$P_{B}(\mathrm{~d})$ & 1161 & $\sim 300^{\dagger}$ & $19(?)$ \\
$v_{w}(\mathrm{~km} / \mathrm{s})$ & 200 & $\sim 300^{\ddagger}$ & 350 \\
$\mu_{30}$ & $?$ & $?$ & 1.7 \\
$\dot{M}_{16}$ & 1 & 0.6 & 0.2 \\
$\dot{\omega}_{s d}^{*}$ & $-2.34 \cdot 10^{-11}-1.63 \cdot 10^{-11}$ & $-9.4 \cdot 10^{-14}$ \\
\hline \multicolumn{4}{c}{} \\
\hline$K_{1}\left(u_{c} / u_{f f}\right) \zeta^{-3 / 11}(1-z / Z)$ & Derived parameters \\
$\mu_{30, \min }^{\prime \prime}$ & $\approx 2.4$ & $\approx 10$ \\
\hline
\end{tabular}

$\dagger$ Estimate of the source’s position in the Corbet diagram ${ }^{\ddagger}$ Estimate of typical wind velocity binary pulsars containing Be-stars.

At very small accretion rates $y \ll 1$ the spin-up torque $K_{s u}$ can be neglected, and the spin-down rate of a pulsar is

$$
\dot{\omega}_{s d}^{*} \approx-0.54 \times 10^{-12}\left[\mathrm{rad} / \mathrm{s}^{2}\right](1-z / Z) K_{1}\left(\frac{u_{c}}{u_{f f}}\right) \zeta^{-3 / 11} \mu_{30}^{13 / 11} \dot{M}_{16}^{3 / 11}\left(\frac{P^{*}}{100 \mathrm{~s}}\right)^{-1}
$$

From this we obtain a lower limit on the neutron star magnetic field that does not depend on the stellar wind velocity and the binary orbital period:

$$
\mu_{30}>\mu_{30, \text { min }}^{\prime \prime} \approx 1.68\left|\frac{\dot{\omega}_{s d}^{*}}{10^{-12} \mathrm{rad} / \mathrm{s}^{2}}\right|^{11 / 13}(1-z / Z)^{-11 / 13}\left[K_{1}\left(\frac{u_{c}}{u_{f f}}\right)\right]^{-11 / 13} \zeta^{3 / 13} \dot{M}_{16}^{-3 / 13}\left(\frac{P^{*}}{100 \mathrm{~s}}\right)^{11 / 13}
$$


As an example, consider the steady spin-down behavior in several slowly rotating moderateluminosity X-ray pulsars (GX 1+4, SXP 1062, 4U 2206+54) within the framework of quasispherical settling accretion theory. The results are summarized in Table 2.

\section{Bright flares in supergiant fast X-ray transients}

Supergiant Fast X-ray Transients (SFXTs) are a subclass of HMXBs associated with early-type supergiant companions $[24,25,26]$, and characterized by sporadic, short and bright $\mathrm{X}$-ray flares reaching peak luminosities of $10^{36}-10^{37} \mathrm{erg} \mathrm{s}^{-1}$. Most of them were discovered by INTEGRAL $[27,28,29,30,31]$. They show high dynamic ranges (between 100 and 10,000, depending on the specific source; e.g. $[32,33])$ and their X-ray spectra in outburst are very similar to accreting pulsars in HMXBs. In fact, half of them have measured neutron star (NS) spin periods similar to those observed from persistent HMXBs (see [34] for a review).

The physical mechanism driving their transient behavior, related to the accretion by the compact object of matter from the supergiant wind, has been discussed by several authors and is still a matter of debate, as some of them require particular properties of the compact objects hosted in these systems $[35,36]$, and others assume peculiar clumpy properties of the supergiant winds and/or orbital characteristics [37, 38, 39, 40, 41, 42].

The typical energy released in a SFXT bright flare is about $10^{38}-10^{40}$ ergs [43], varying by one order of magnitude between different sources. That is, the mass fallen onto the NS in a typical bright flare varies from $10^{18} \mathrm{~g}$ to around $10^{20} \mathrm{~g}$.

The typical X-ray luminosity outside outbursts in SFXTs is about $L_{x, \text { low }} \simeq 10^{34} \mathrm{erg} \mathrm{s}^{-1}$ [44], and below we shall normalise the luminosity to this value, $L_{34}$. At these low X-ray luminosities, the plasma entry rate into the magnetosphere is controlled by radiative plasma cooling. Further, it is convenient to normalise the typical stellar wind velocity from hot OB-supergiants $v_{w}$ to $1000 \mathrm{~km} \mathrm{~s}^{-1}$ (for orbital periods of about a few days or larger the NS orbital velocities can be neglected compared to the stellar wind velocity from the OB-star), so that the Bondi gravitational capture radius is $R_{B}=2 G M / v_{w}^{2}=4 \times 10^{10}[\mathrm{~cm}] v_{8}^{-2}$ for a fiducial NS mass of $M_{x}=1.5 M_{\odot}$.

\subsection{Magnetopsheric shell instability}

Let us assume that a quasi-static shell hangs over the magnetosphere around the NS, with the magnetospheric accretion rate being controlled by radiative plasma cooling. We denote the actual steady-state accretion rate as $\dot{M}_{a}$ so that the observed X-ray steady-state luminosity is $L_{x}=$ $0.1 \dot{M}_{a} c^{2}$. Then from the theory of subsonic quasi-spherical accretion [14] we know that the factor $f(u)$ (the ratio of the actual velocity of plasma entering the magnetosphere, due to the RayleighTaylor instability, to the free-fall velocity at the magnetosphere, $\left.u_{f f}\left(R_{A}\right)=\sqrt{2 G M / R_{A}}\right)$ reads $[45$, 46]

$$
f(u)_{\text {rad }} \simeq 0.036 \zeta^{7 / 11} L_{34}^{2 / 9} \mu_{30}^{2 / 27}
$$

(See also Eq. (7.1) above).

The shell is quasi-static (and likely convective). It is straightforward to calculate the mass of the shell using the density distribution $\rho(R) \propto R^{-3 / 2}$ [14]. Using the mass continuity equation to 
eliminate the density above the magnetosphere, we readily find

$$
\Delta M \approx \frac{2}{3} \frac{\dot{M}_{a}}{f(u)} t_{f f}\left(R_{B}\right)
$$

Note that this mass can be expressed through measurable quantities $L_{x, l o w}, \mu_{30}$ and the (not directly observed) stellar wind velocity at the Bondi radius $v_{w}\left(R_{B}\right)$. Using Eq. (7.1) for the radiative plasma cooling, we obtain

$$
\Delta M_{\text {rad }} \approx 8 \times 10^{17}[g] \zeta^{-7 / 11} L_{34}^{7 / 9} v_{8}^{-3} \mu_{30}^{-2 / 27} .
$$

The simple estimate (7.3) shows that for a typical wind velocity near the NS of about $500 \mathrm{~km} \mathrm{~s}^{-1}$ the typical mass of the hot magnetospheric shell is around $10^{19} \mathrm{~g}$, corresponding to $10^{39} \mathrm{ergs}$ released in a flare if all the matter from the shell is accreted onto the NS, as observed. Variations in stellar wind velocity between different sources by a factor of $\sim 2$ would produce the one-order-ofmagnitude spread in $\Delta M$ observed in bright SFXT flares.

As noted in [45], if there is an unstable matter flow through the magnetosphere, a large quantity of X-ray photons produced near the NS surface should rapidly cool down the plasma near the magnetosphere, further increasing the plasma fall velocity $u_{R}\left(R_{A}\right)$ and the ensuing accretion NS luminosity $L_{x}$. Therefore, in a bright flare the entire shell can fall onto the NS on the free-fall time scale from the outer radius of the shell $t_{f f}\left(R_{B}\right) \sim 1000 \mathrm{~s}$. Clearly, the shell will be replenished by new wind capture, so the flares will repeat as long as the rapid mass entry rate into the magnetosphere is sustained.

\subsection{Magnetized stellar wind as the flare trigger}

We suggest that the shell instability described above can be triggered by a large-scale magnetic field sporadically carried by the stellar wind of the optical OB companion. Observations suggest that about $\sim 10 \%$ of hot OB-stars have magnetic fields up to a few $\mathrm{kG}$ (see [47] for a review and discussion). It is also well known from Solar wind studies (see e.g. reviews [48, 49] and references therein) that the Solar wind patches carrying tangent magnetic fields has a lower velocity (about $350 \mathrm{~km} \mathrm{~s}^{-1}$ ) than the wind with radial magnetic fields (up to $\sim 700 \mathrm{~km} \mathrm{~s}^{-1}$ ). Fluctuations of the stellar wind density and velocity from massive stars are also known from spectroscopic observations [50], with typical velocity fluctuations up to $0.1 v_{\infty} \sim 200-300 \mathrm{~km} \mathrm{~s}^{-1}$.

The effect of the magnetic field carried by the stellar wind is twofold: first, it may trigger rapid mass entry to the magnetosphere via magnetic reconnection (the phenomenon well known in the dayside Earth magnetosphere, [51]), and secondly, the magnetized parts of the wind (magnetized clumps with a tangent magnetic field) have a lower velocity than the non magnetized ones (or the ones carrying the radial field). As discussed in [43] and below, magnetic reconnection can increase the plasma fall velocity in the shell from inefficient, radiative-cooling controlled settling accretion with $f(u)_{\text {rad }} \sim 0.03-0.1$, up to the maximum possible free-fall velocity with $f(u)=1$. In other words, during a bright flare subsonic settling accretion turns into supersonic Bondi accretion. The second factor (slower wind velocity in magnetized clumps with tangent magnetic field) strongly increases the Bondi radius $R_{B} \propto v_{w}^{-2}$ and the corresponding Bondi mass accretion rate $\dot{M}_{B} \propto v_{w}^{-3}$.

Indeed, we can write down the mass accretion rate onto the NS in the unflaring (low-luminosity) state as $\dot{M}_{a, l o w}=f(u) \dot{M}_{B}$ with $f(u)$ given by expression (7.1) and $\dot{M}_{B} \simeq \pi R_{B}^{2} \rho_{w} v_{w}$. Eliminating the 
wind density $\rho_{w}$ using the mass continuity equation written for the spherically symmetric stellar wind from the optical star with power $\dot{M}_{o}$ and assuming a circular binary orbit, we arrive at $\dot{M}_{B} \simeq \frac{1}{4} \dot{M}_{o}\left(\frac{R_{B}}{a}\right)^{2}$. Using the well-known relation for the radiative wind mass-loss rate from massive hot stars $\dot{M}_{o} \simeq \epsilon \frac{L}{c v_{\infty}}$ where $L$ is the optical star luminosity, $v_{\infty}$ is the stellar wind velocity at infinity, typically $2000-3000 \mathrm{~km} \mathrm{~s}^{-1}$ for OB stars and $\epsilon \simeq 0.4-1$ is the efficiency factor [52] (in the numerical estimates below we shall assume $\epsilon=0.5)$. It is also possible to reduce the luminosity $L$ of a massive star to its mass $M$ using the phenomenological relation $\left(L / L_{\odot}\right) \approx 19\left(M / M_{\odot}\right)^{2.76}$ (see e.g. [53]). Combining the above equations and using Kepler's third law to express the orbital separation $a$ through the binary period $P_{b}$, we find for the X-ray luminosity of SFXTs in the non-flaring state

$$
\begin{aligned}
L_{x, \text { low }} \simeq & 5 \times 10^{35}\left[\mathrm{erg} \mathrm{s}^{-1}\right] f(u)\left(\frac{M}{10 M_{\odot}}\right)^{2.76-2 / 3} \\
& \left(\frac{v_{\infty}}{1000 \mathrm{~km} \mathrm{~s}^{-1}}\right)^{-1}\left(\frac{v_{w}}{500 \mathrm{~km} \mathrm{~s}^{-1}}\right)^{-4}\left(\frac{P_{b}}{10 \mathrm{~d}}\right)^{-4 / 3},
\end{aligned}
$$

which for $f(u) \sim 0.03-0.1$ corresponds to the typical low-state luminosities of SFXTs of $\sim 10^{34} \mathrm{erg} \mathrm{s}^{-1}$.

It is straightforward to see that a transition from the low state (subsonic accretion with slow magnetospheric entry rate $f(u) \sim 0.03-0.1$ ) to supersonic free-fall Bondi accretion with $f(u)=1$ due to the magnetized stellar wind with the velocity decreasing by a factor of two, for example, would lead to a flaring luminosity of $L_{x, \text { flare }} \sim(10 \div 30) \times 2{ }^{5} L_{x, l o w}$. This shows that the dynamical range of SFXT bright flares $(\sim 300-1000)$ can be naturally reproduced by the proposed mechanism.

\subsection{Conditions for magnetic reconnection near the magnetosphere}

For magnetic field reconnection to occur, the time the magnetized plasma spends near the magnetopause should be at least comparable to the reconnection time, $t_{r} \sim R_{A} / v_{r}$, where $v_{r}$ is the magnetic reconnection rate, which is difficult to assess from first principles [54]. In real astrophysical plasmas the large-scale magnetic reconnection rate can be as high as $v_{r} \sim 0.03-0.07 v_{A}$ [54], and phenomenologically we can parametrize it as $v_{r}=\epsilon_{r} v_{A}$ with $\epsilon_{r} \sim 0.01-0.1$. The longest time-scale the plasma penetrating into the magnetosphere spends near the magnetopause is the instability time, $t_{\text {inst }} \sim t_{f f}\left(R_{A}\right) f(u)_{\text {rad }}$ [14], so the reconnection may occur if $t_{r} / t_{\text {inst }} \sim\left(u_{f f} / v_{A}\right)\left(f(u)_{\text {rad }} / \epsilon_{r}\right) \lesssim 1$. As near $R_{A}$ (from its definition) $v_{A} \sim u_{f f}$, we arrive at $f(u)_{\text {rad }} \lesssim \epsilon_{r}$ as the necessary reconnection condition. According to Eq. (7.1), it is satisfied only at sufficiently low X-ray luminosities, pertinent to 'quiet' SFXT states. This explains why in HMXBs with convective shells at higher luminosity (but still lower than $4 \times 10^{36} \mathrm{erg} \mathrm{s}^{-1}$, at which settling accretion is possible), reconnection from magnetized plasma accretion will not lead to the shell instability, but only to temporal establishment of the 'strong coupling regime' of angular momentum transfer through the shell, as discussed in [14]. Episodic strong spin-ups, as observed in GX 301-2, may be manifestations of such 'failed' reconnection-induced shell instability.

Therefore, it seems likely that the key difference between steady HMXBs like Vela X-1, GX 301-2 (showing only moderate flaring activity) and SFXTs is that in the first case the effects of possibly magnetized stellar winds from optical OB-companions are insignificant (basically due to the rather high mean accretion rate), while in SFXTs with lower 'steady' X-ray luminosity, largescale magnetic fields, sporadically carried by clumps in the wind, can trigger SFXT flaring activity 
via magnetic reconnection near the magnetospheric boundary. The observed power-law SFXT flare distributions, discussed in [55], with respect to the log-normal distributions for classical HMXBs [56], may be related to the properties of magnetized stellar wind and physics of its interaction with the NS magnetosphere $[57,58]$.

Acknowledgement. The work is supported by the Russian Science Foundation grant 14-1200146.

\section{References}

[1] R. Giacconi, H. Gursky, F. R. Paolini, and B. B. Rossi, Physical Review Letters 9, 439 (1962).

[2] J. H. M. M. Schmitt, S. L. Snowden, B. Aschenbach, G. Hasinger, E. Pfeffermann, P. Predehl, and J. Trumper, Nature 349, 583 (1991).

[3] R. Giacconi, E. Kellogg, P. Gorenstein, H. Gursky, and H. Tananbaum, ApJL 165, L27 (1971).

[4] Y. B. Zel'dovich, Doklady Akademii Nauk SSSR 155, 67 (1964).

[5] E. E. Salpeter, ApJ 140, 796 (1964).

[6] M. Oda, P. Gorenstein, H. Gursky, E. Kellogg, E. Schreier, H. Tananbaum, and R. Giacconi, ApJL 166, L1 (1971).

[7] R. Giacconi, H. Gursky, E. Kellogg, E. Schreier, and H. Tananbaum, ApJL 167, L67 (1971).

[8] H. Tananbaum, H. Gursky, E. M. Kellogg, R. Levinson, E. Schreier, and R. Giacconi, ApJL 174, L143 (1972).

[9] N. I. Shakura and R. A. Sunyaev, A\&A 24, 337 (1973).

[10] K. Davidson and J. P. Ostriker, ApJ 179, 585 (1973).

[11] J. Arons and S. M. Lea, ApJ 207, 914 (1976).

[12] A. F. Illarionov and R. A. Sunyaev, A\&A 39, 185 (1975).

[13] D. J. Burnard, J. Arons, and S. M. Lea, ApJ 266, 175 (1983).

[14] N. Shakura, K. Postnov, A. Kochetkova, and L. Hjalmarsdotter, MNRAS 420, 216 (2012).

[15] R. E. Davies and J. E. Pringle, MNRAS 196, 209 (1981).

[16] R. F. Elsner and F. K. Lamb, ApJ 215, 897 (1977).

[17] A. S. Kompaneets, Zh. Eksp. Theor. Phys. 31, 876 (1956).

[18] R. Weymann, Physics of Fluids 8, 2112 (1965).

[19] N. I. Shakura, K. A. Postnov, A. Y. Kochetkova, and L. Hjalmarsdotter, Physics-Uspekhi 56, 321 (2013).

[20] N. R. Ikhsanov, Y. S. Likh, and N. G. Beskrovnaya, Astronomy Reports 58, 376 (2014).

[21] V. Doroshenko, A. Santangelo, V. Suleimanov, I. Kreykenbohm, R. Staubert, C. Ferrigno, and D. Klochkov, A\&A 515, A10 (2010).

[22] N. I. Shakura, K. A. Postnov, A. Y. Kochetkova, and L. Hjalmarsdotter, Physics Uspekhi 56, 321-346 (2013). 
[23] L. I. Sedov, Similarity and Dimensional Methods in Mechanics (1959).

[24] L. J. Pellizza, S. Chaty, and I. Negueruela, A\&A 455, 653 (2006).

[25] S. Chaty, F. Rahoui, C. Foellmi, J. A. Tomsick, J. Rodriguez, and R. Walter, A\&A 484, 783 (2008).

[26] F. Rahoui, S. Chaty, P.-O. Lagage, and E. Pantin, A\&A 484, 801 (2008).

[27] S. Molkov, N. Mowlavi, A. Goldwurm, A. Strong, N. Lund, J. Paul, and T. Oosterbroek, The Astronomer's Telegram 176, 1 (2003).

[28] R. A. Sunyaev, S. A. Grebenev, A. A. Lutovinov, J. Rodriguez, S. Mereghetti, D. Gotz, and T. Courvoisier, The Astronomer's Telegram 190, 1 (2003).

[29] S. A. Grebenev, A. A. Lutovinov, and R. A. Sunyaev, The Astronomer's Telegram 192, 1 (2003).

[30] V. Sguera, E. J. Barlow, A. J. Bird, D. J. Clark, A. J. Dean, A. B. Hill, L. Moran, S. E. Shaw, D. R. Willis, A. Bazzano, P. Ubertini, and A. Malizia, A\&A 444, 221 (2005).

[31] I. Negueruela, D. M. Smith, P. Reig, S. Chaty, and J. M. Torrejón, in Proc. of the "The X-ray Universe 2005”, Ed. by A. Wilson. ESA SP-604, Vol. 1, 2006 (2006), 165.

[32] P. Romano, V. La Parola, S. Vercellone, G. Cusumano, L. Sidoli, H. A. Krimm, C. Pagani, P. Esposito, E. A. Hoversten, J. A. Kennea, K. L. Page, D. N. Burrows, and N. Gehrels, MNRAS 410, 1825 (2011).

[33] P. Romano, H. A. Krimm, D. M. Palmer, L. Ducci, P. Esposito, S. Vercellone, P. A. Evans, C. Guidorzi, V. Mangano, J. A. Kennea, S. D. Barthelmy, D. N. Burrows, and N. Gehrels, A\&A 562, A2 (2014).

[34] L. Sidoli, in Proc. 9th INTEGRAL Workshop. Published online at "http://pos.sissa.it/cgi-bin/reader/conf.cgi?confid=176", id.11 (2012).

[35] S. A. Grebenev and R. A. Sunyaev, Astronomy Letters 33, 149 (2007).

[36] E. Bozzo, M. Falanga, and L. Stella, ApJ 683, 1031 (2008).

[37] J. J. M. in't Zand, A\&A 441, L1 (2005).

[38] R. Walter and J. Zurita Heras, A\&A 476, 335 (2007).

[39] L. Sidoli, P. Romano, S. Mereghetti, A. Paizis, S. Vercellone, V. Mangano, and D. Götz, A\&A 476, 1307 (2007).

[40] I. Negueruela, J. M. Torrejón, P. Reig, M. Ribó, and D. M. Smith, in AIP Conf. Ser., vol. 1010 (Edited by R. M. Bandyopadhyay, S. Wachter, D. Gelino, and C. R. Gelino) (2008), vol. 1010, 252-256.

[41] L. Ducci, L. Sidoli, S. Mereghetti, A. Paizis, and P. Romano, MNRAS 398, 2152 (2009).

[42] L. M. Oskinova, A. Feldmeier, and P. Kretschmar, MNRAS 421, 2820 (2012).

[43] N. Shakura, k. Postnov, L. Sidoli, and A. Paizis, ArXiv e-prints (2014).

[44] L. Sidoli, P. Romano, V. Mangano, A. Pellizzoni, J. A. Kennea, G. Cusumano, S. Vercellone, A. Paizis, D. N. Burrows, and N. Gehrels, ApJ 687, 1230 (2008).

[45] N. Shakura, K. Postnov, and L. Hjalmarsdotter, MNRAS 428, 670 (2013).

[46] N. I. Shakura, K. A. Postnov, A. Y. Kochetkova, and L. Hjalmarsdotter, in European Physical Journal Web of Conferences, European Physical Journal Web of Conferences, vol. 64 (2014), European Physical Journal Web of Conferences, vol. 64, 2001. 
[47] J. Braithwaite, ArXiv e-prints (2013).

[48] L. M. Zelenyi and A. V. Milovanov, Physics Uspekhi 47, 1 (2004).

[49] R. Bruno and V. Carbone, Living Reviews in Solar Physics 10 (2013).

[50] J. Puls, J. S. Vink, and F. Najarro, A\&A Review16, 209 (2008).

[51] J. W. Dungey, Physical Review Letters 6, 47 (1961).

[52] H. J. G. L. M. Lamers, E. P. J. van den Heuvel, and J. A. Petterson, A\&A 49, 327 (1976).

[53] E. A. Vitrichenko, D. K. Nadyozhin, and T. L. Razinkova, Astronomy Letters 33, 251 (2007).

[54] E. G. Zweibel and M. Yamada, ARAA 47, 291 (2009).

[55] A. Paizis and L. Sidoli, MNRAS 439, 3439 (2014).

[56] F. Fürst, I. Kreykenbohm, K. Pottschmidt, J. Wilms, M. Hanke, R. E. Rothschild, P. Kretschmar, N. S. Schulz, D. P. Huenemoerder, D. Klochkov, and R. Staubert, A\&A 519, A37 (2010).

[57] N. Shakura, K. Postnov, L. Sidoli, and A. Paizis, MNRAS 442, 2325 (2014).

[58] L. Sidoli, A. Paizis, and K. Postnov, MNRAS 457, 3693 (2016). 